\title{
Cemento aluminoso: cómo evitar la pérdida de las resistencias mecánicas
}

\section{Ciment fondu: Comme eviter leur dégradation des résistences mécaniques}

Fecha de recepción: 15/V/87

\section{RESUMEN}

Para evitar la degradación de la resistencia mecánica producida por la conversión del hexagonal al cúbico en el cemento aluminoso se han estudiado diversos procesos.

1) Tratamiento térmico durante el fraguado de una mezcla de cemento aluminoso y carbonato cálcico, este proceso utiliza la fase elástica durante el fraguado y también la reacción del carbonato cálcico con la alúmina del cemento para formar carboaluminatos.

2) Tratamientos térmicos con $\mathrm{CO}_{2}$ a $1,4 \mathrm{kp} / \mathrm{cm}^{2}$, en condiciones variables de humedad que aprovechan la fase elástica del fraguado y también la reación del $\mathrm{CO}_{2}$ con la alúmina del cemento aluminoso para formar carboaluminatos con aumentos de la resistencia mecánica.

Se dan las mejores condiciones obtenidas con probetas de mortero silícico de $4 \times 4 \times 16 \mathrm{~cm}$.

\author{
F. TRIVIÑO \\ IETCC/CSIC
}

Serrano Galvache, s.n. 28033 - Madrid/España

\section{RESUME}

Pour éviter la diminution de la résistence mécanique produite par la conversion hexagonal à cubique, dans le ciment fondu différents procédés sont etudiée:

1) Traitament thermique pendant la prise, du ciment fondu et carbonate calcique, qui utilise la phase elastique de la prise et aussi la réaction du carbonate calcique avec l'alumina du ciment fondu pour former carboaluminates.

2) Traitaments thermique avec le $\mathrm{CO}_{2}$ a $1,4 \mathrm{kp} / \mathrm{cm}^{2}$, dans distinctes conditions d'humidité, qui utilisen la phase élastique de la prise et aussi la reaction du le $\mathrm{CO}_{2}$ avec l'alumina du cement fondu pour former carboaluminates avec un accroissement de la résistence mécanique.

Les meilleurs conditions obtenues, avec eprouvettes de mortier silicique de $4 \times 4 \times 16 \mathrm{~cm}$. sont données.

\section{EFECTO DE LA ADICION DE CALCITA AL CEMENTO ALUMINOSO}

\subsection{Experimental}

Con proporciones variables de $\mathrm{CaCO}_{3}$ de $0 \%$, $15 \%, 20 \%$ y cemento aluminoso se hicieron probetas prismáticas de $4 \times 4 \times 16 \mathrm{~cm}$.

Se realizaron tres tipos de tratamiento.

1. Se colocan las probetas en cámara húmeda. Se las desmoldea a las 19 horas de su preparación.

2. Se calientan a $50^{\circ} \mathrm{C}$; el cemento, el agua y moldes. Una vez confeccionados las probetas y los moldes se mantienen a $50^{\circ} \mathrm{C}$ durante 19 horas. Se desmoldean las probetas y se mantienen 19 horas en cámara húmeda.

\section{EFFET DE L'ADDITION DE CALCAIRE AU CIMENT FONDU}

\subsection{Experimental}

Avec des proportions variables de $\mathrm{CaCO}_{3}$ de $0 \%, 15 \%$, et $20 \%$ et du ciment fondu, des éprouvettes prismatiques de $4 \times 4 \times 16 \mathrm{~cm}$ furent confectionnées.

Trois types de traitement furent effectués:

1. Le éprouvettes sont placées en chambre humide. On procède à les démouler 19 heures après leur préparation.

2. Le ciment, l'eau et les moules sont chauffés à $50^{\circ} \mathrm{C}$. Une fois que les éprouvettes et les moules sont confectionnés, ils sont maintenus à $50^{\circ} \mathrm{C}$ pendant 19 heures. On procède à démouler les éprouvettes, qui sont placées pendant 24 heures en chambre humide. 
3. Las probetas desmoldeadas que se mantuvieron a $50^{\circ} \mathrm{C}$ se introdujeron en un recipiente hermético (fig. 1) a $50^{\circ} \mathrm{C}$ y se trataron con $\mathrm{CO}_{2}$ a una presión de $0,14 \mathrm{~N}$ durante 5 horas. Terminado este tiempo se introdujeron en cámara húmeda durante 19 horas.

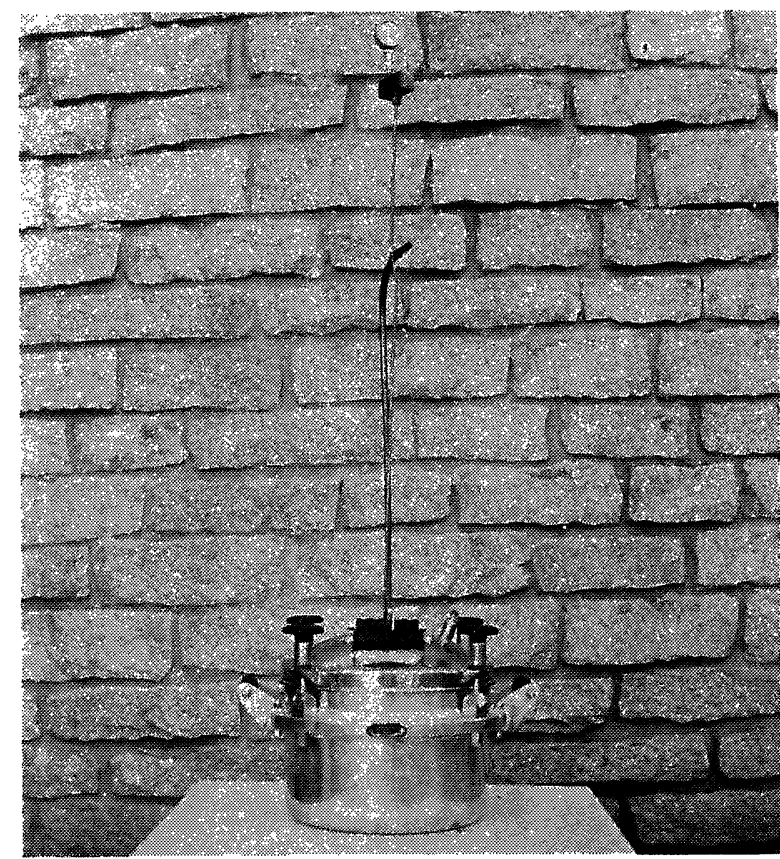

Fig. 1

\subsection{Resultados}

Los resultados de la rotura a compresión y flexión, así como los obtenidos de los difractogramas de rayos $X$, de las intensidades relativas de los compuestos: $\mathrm{CA}, \mathrm{CAH}_{10}$, $\mathrm{C}_{3} \mathrm{AH}_{6}, \mathrm{AH}_{3}, \mathrm{CC}$ y $\mathrm{cC}_{4} \mathrm{AH}_{12}$ se utilizaron para realizar las gráficas de la fig. 2 .

\subsection{Interpretación y Conclusiones}

1. La resistencia a compresión con los tres tratamientos aumenta si lo hace el $\mathrm{CaCO}_{3}$.

3. La resistencia a compresión aumenta para el $20 \%$ de $\mathrm{CaCO}_{3}$, al realizar el tratamiento térmico y con $\mathrm{CO}_{2}$.
3. Le éprouvettes démoulées, qui étaient restées à $50^{\circ} \mathrm{C}$ son introduites dans un récipient hermétique (fig. 1) à $50^{\circ} \mathrm{C}$ et sont traitées avec $\mathrm{CO}_{2}$ à une pression de $0,14 \mathrm{~N}$ pendant 5 heures. Ce temps ayant pris fin, elles sont introduites en chambre humide pendant 19 heures.

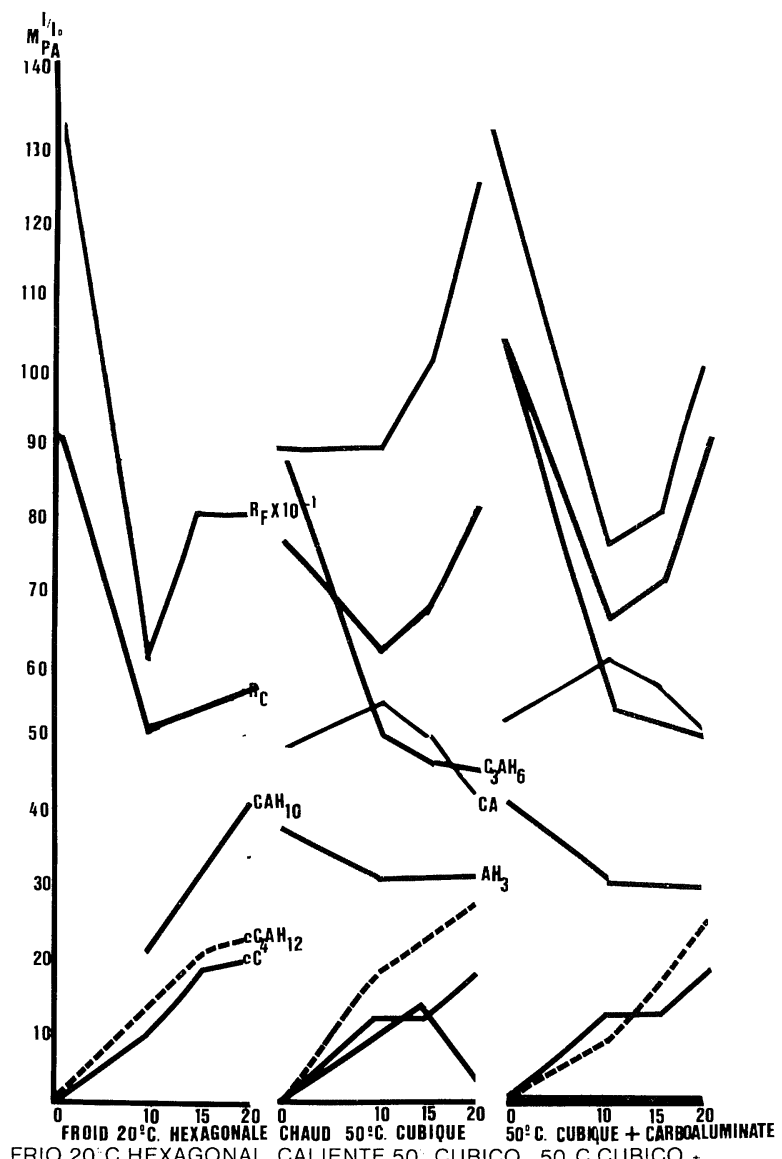
FRIO 20 C HEXAGONAL. CALIENTE 50 CUBICO. 50 C CUBICO +

Fig. 2

\subsection{Resultats}

Les résultats de rupture à compression et à flexion, ainsi que ceux obtenus des intensités relatives des composés: $C A, C A H_{10}, C_{3} A H_{6}$, $\mathrm{AH}_{3}, \mathrm{CC}, \mathrm{CC}_{4} \mathrm{AH}_{12}$ sont utilisés pour confectionner les graphiques de la figure 2.

\subsection{Interpretation et Conclusions}

1. La résistance à compresion, dans les trois traittements, augmente si le contenu de $\mathrm{CaCO}_{3}$ augmente.

2. La résistance à compression augmente pour $20 \%$ de $\mathrm{CaCO}_{3}$, en effectuant les traitments thermique et de $\mathrm{CO}_{2}$.

MATERIALES DE COSTRUCCION, Vol. 37, n.o 206, abril/mayo/junio 1987 
3. Al realizar el tratamiento térmico hay una disminución de la resistencia a compresión - cuando no hay adición de calcita- a causa de la reacción $\mathrm{CA} \rightarrow \mathrm{CAH}_{10} \rightarrow \mathrm{C}_{3} \mathrm{AH}_{6}$, disminuyendo el $\mathrm{CAH}_{10}$ y el $\mathrm{CA}$, y aumenta la resistencia con el $\mathrm{CO}_{2}$, en el paso de $\mathrm{C}_{3} \mathrm{AH}_{6} \rightarrow \mathrm{CC}_{4} \mathrm{AH}_{12}$ y a otros carboaluminatos cálcicos hidratados.

4. La concentración de $\mathrm{AH}_{3}$ se mantiene en los difractogramas - lo que indica un aumento proporcional- si aumenta la cantidad de $\mathrm{CaCO}_{3}$ desde $10 \%, 15 \%$ y $20 \%$ respectivamente.

5. Si el tratamiento se realiza a $50^{\circ} \mathrm{C}$ con $\mathrm{CO}_{2}$, disminuye en los difractogramas la concentración detectada de $\mathrm{CaCO}_{3}$, lo que indica que cuando las muestras se calientan, el $\mathrm{CaCO}_{3}$ reacciona con los aluminatos, por lo que solamente con el tratamiento térmico y sin tratamiento con $\mathrm{CO}_{2}$ se obtienen carboaluminatos cálcicos hidratados y por lo tanto hay un aumento de las resistencias mecánicas.

Las resistencias a flexión disminuyen con el tratamiento térmico y tienden a recuperararse por carbonatación con $\mathrm{CO}_{2}$, lo que indica que el tipo de cristales producidos por el tratamiento (pentágonos dodecaedros de $\mathrm{C}_{3} \mathrm{AH}_{6}$ ) tienen menor resistencia a flexión, que los hexagonales de $\mathrm{CAH}_{10}$, producidos por el tratamiento a $20^{\circ} \mathrm{C}$, y también que los cristales de carboaluminato cálcico hidratado, bajo forma de agujas (provocadas por la reacción con el $\mathrm{CaCO}_{3} \mathrm{O}$ con el $\mathrm{CO}_{2}$ ) favorecen las resistencias a flexión. Finalmente cuando se forman cristales de calcita (romboedros) disminuyen nuevamente las resistencias a flexión.

Por esto las resistencias a flexión aumentan por tratamiento térmico en caliente, cuando aumenta la concentración de $\mathrm{CaCO}_{3}$ en la mezcla y/o si se tratan las mezclas más ricas en $\mathrm{CaCO}_{3}$ con $\mathrm{CO}_{2}$ (efecto sinérgico) por formación de carboaluminatos.

\section{ESTUDIO DEL TIEMPO DE TRATAMIENTO CON ADICION DE CALCITA}

\subsection{Experimental}

Comparación entre los dos tipos de tratamiento.

1. Probetas prismáticas de $4 \times 4 \times 16 \mathrm{~cm}$
3. Il y a, à réaliser le traitement thermique, une diminution de la résistance à compression -lorsqu'il n'y a pas d'adition de calcite- à cause de la reaction de $\mathrm{CA} \rightarrow \mathrm{CAH}_{10} \rightarrow$ $\rightarrow \mathrm{C}_{3} \mathrm{AH}_{6}$, diminuant le $\mathrm{CAH}_{10}$ et le $\mathrm{CA}$, qui remonte au moyen d'un traitement avec $\mathrm{CO}_{2}$ avec passage partiel de $\mathrm{C}_{3} \mathrm{AH}_{6} \rightarrow \mathrm{CC}_{4} \mathrm{AH}_{12}$ et autres carboaluminates calciques hydratés.

4. La concentration de $\mathrm{AH}_{3}$ se maintient dans les difractogrammes - ce qui indique le croissance proportionnelle- si la quantité de $\mathrm{CaCO}_{3}$ augmente, c'est à dire, 10, 15 et $20 \%$ respectivement.

5. Si on effectue les traitements à $50^{\circ} \mathrm{C}$ avec $\mathrm{CO}_{2}$, la concentration de $\mathrm{CaCO}_{3}$ détectée dans les difractogrammes diminue, ce qui indique que lorsque les échantillons sont chauffés, le $\mathrm{CaCO}_{3}$ réactionne avec les aluminates, c'est pourquoi, rien qu'avec le traitement thermique, on obtient déjà la formation des carboaluminates calciques hydratés sans qu'il soit nécessaire d'ajouter $\mathrm{CO}_{2}$, et en conquence on obtient l'augmentation des résistances mécaniques.

Las résistances à flexion diminuet avec le traitement thermique et tendent à se récupérer par carbonatation avec $\mathrm{CO}_{2}$, ce qui indique que le type de cristaux produits par le traitement thermique (pentagones dodécaèdres du $\mathrm{C}_{3} \mathrm{AH}_{6}$ ) offre une plus petite résistance à la flexion que les hexagonels du $\mathrm{CAH}_{10}$, produits par le traitement à $20^{\circ} \mathrm{C}$, et aussi que les cristaux de carboaluminate calcique hydratés sous forme d'aiguilles (provoqués par la réaction avec le $\mathrm{CaCO}_{3}$ ou avec le $\mathrm{CO}_{2}$ ) que favorisent les résistances à flexion. Et finalement, lorsque des cristaux de calcite se forment (rhomboèdres), les résistances à flexion diminuent à nouveau. De cette façon les résistances à flexión augmentent par traitement thermique au chaud lorsque la concentration de $\mathrm{CaCO}_{3}$ augmente dans le mélange et/ou si l'on traite les mélanges plus riches en $\mathrm{CaCO}_{3}$ avec $\mathrm{CO}_{2}$ (effect synergique) par formation de carboaluminates.

\section{ETUDE DU TEMPS DE TRAITEMENT AVEC ADITION DE CALCITE}

\subsection{Experimental}

Comparaison entre deux types de traitement.

1. Les éprouvettes prismatiques de $4 \times 4 \times 16$ 
realizadas con cemento aluminoso y $20 \%$ de $\mathrm{CaCO}_{3}$, con una relación agua/cemento de $0,23 \%$ tratados a $20^{\circ} \mathrm{C}$ en cámara húmeda durante 2,4 y 14 días.

2. Probetas iguales a las del punto 1 , curadas a $50^{\circ} \mathrm{C}$ con $\mathrm{CO}_{3}$ a 2, 3, 4, 6, 9 y 14 días.

\subsection{Resultados}

Los resultados se pueden ver en la figura 3. En los dos casos se observa un aumento de las resistencias a flexión y a compresión superiores a las de los primeros casos y una caida en las resistencias a flexión entre 9 y 14 días, debidas al paso de carboaluminatos, a carbonato cálcico, por descomposición de los carboaluminatos con el carbónico. De lo anterior se puede deducir que la formación por meteorización de carbonato cálcico implica una recuperación de las resistencias iniciales a flexión y un aumento de las resistencias a compresión. cm confectionnées avec ciment alumineux et $20 \%$ de $\mathrm{CaCO}_{3}$, avec une relation eau-ciment de $0,235 \%$, traitées à $20^{\circ} \mathrm{C}$ en chambre humide pendant 2, 4 et 14 jours.

2. Eprouvettes égales aux premières, curées à $50^{\circ} \mathrm{C}$ avec $\mathrm{CO}_{2}$, à 2, 3, 4, 6, 9 et 14 jours.

\subsection{Resultats}

Les résultats apparaissent dans la figure 3.

Dans les deux cas on observe une augmentation des résistance à flexion et à compression avec un temps supérieur aux précédents et une chute dans les résistances à flexion entre 9 et 14 jours, dues u passage de carboaluminates à carbonate calcique, par décomposition des précedents avec le carbonique. De ce qui vient d'être dit, on peut déduire que la formation de carbonate calcique par météorisation implique une récupération des résistances à flexion initiales et une montée des résistances à compression.

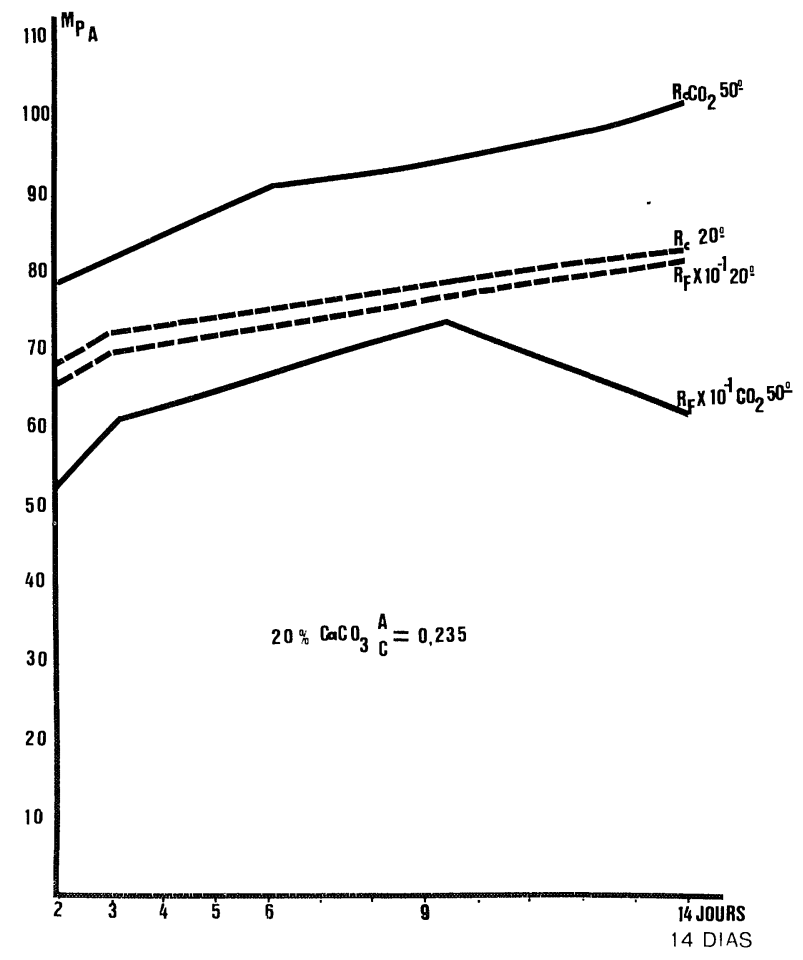

Fig. 3

\section{MORTEROS SILICICOS}

\subsection{Preparación de las probetas}

En una amasadora normalizada de acuerdo con el RC-75, se mezclaron: 1.500 gramos de arena silícica normalizada, 500 gramos de cemento aluminoso y $214 \mathrm{ml}$ de agua

\section{MORTIER SILICIQUES}

\subsection{Preparation des eprouvettes}

Dans un malaxeur normalisé, d'après le $R C-75$, $1.500 \mathrm{gr}$ de sable silicique normalisé, $500 \mathrm{gr}$ de ciment alumineux et $214 \mathrm{ml}$ d'eau déionisée furent mélangés. Une partie du mortier fut 
desionizada. Una parte del mortero se colocó en un molde, con tres improntas prismáticas de $4 \times 4 \times 16 \mathrm{~cm}$, situado sobre una máquina de sacudidas normalizadas según el RC-75.

\subsection{Tratamiento de las probetas}

El molde cubierto con una lámina plástica (para evitar el enarenado superficial) se colocó en un recipiente a presión fig. 1, en el cual se realizaron los tratamientos en caliente o a temperatura ambiente con $\mathrm{CO}_{2}$.

Los moldes y también las probetas desmoldeadas, se separaron con listoncillos, para evitar su contacto y también con el agua, que se encontraba en algunas ocasiones en el fondo del recipiente. La tapadera del recipiente a presión tiene unos cierres de tornillo rotativo, de seguridad, que se aflojan para retirar la tapa.

En la tapadera hay un tubo para entrada del gas y un manómetro (fig. 1).

Los tratamientos realizados con las probetas de mortero aluminoso fueron los siguientes: placée dans un moule, avec trois empreintes prismatiques de $4 \times 4 \times 16 \mathrm{~cm}$ situé sur une machine à secousses normalisée, d'après le RC-75.

\subsection{Le traitement des eprouvettes}

Le moule couvert avec une lame en plastique (pour éviter l'ensblement superficiel) est placé dans un autocuiseur (fig. 1) où sont effectués les traitements au chaud ou à température ambiante avec $\mathrm{CO}_{2}$. Les moules, ou seulement les éprouvettes démoulées sont séparés avec des liteaux, pour éviter le contact entre eux et avec l'eau qui se trouve quelques fois au fond de l'autocuiseur. Le couvercle de l'autocuiseur a une soupape de sûreté qu'il fut ouvrir pour retirer.

Dans le couvercle il y a aussi un tuyau pour l'accès des gaz, et un manomètre (fig. 1).

Les traitements effectués avec les éprouvettes de mortier alumineux on été les suivants:

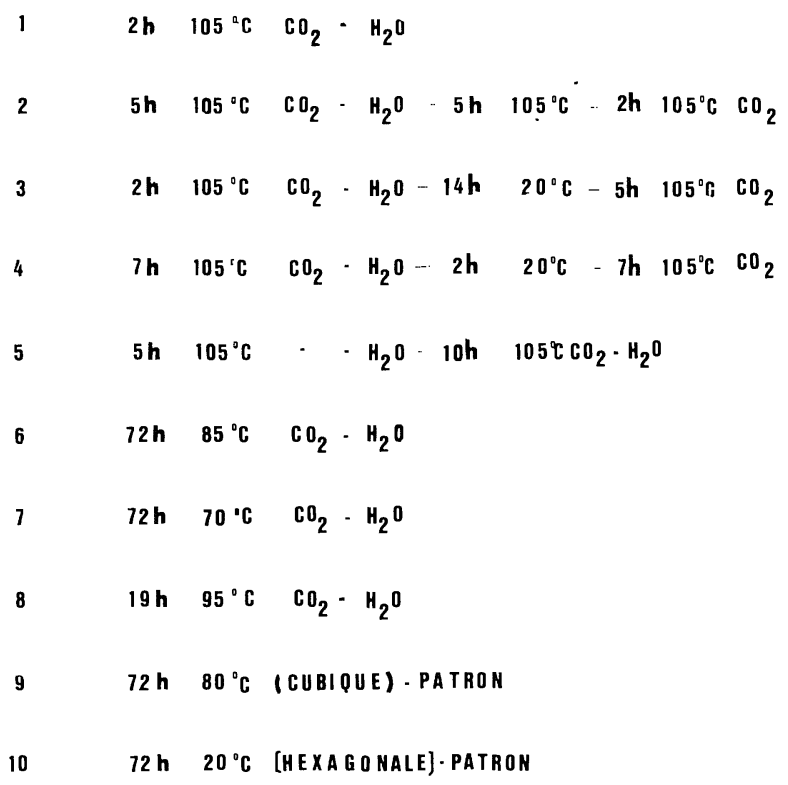

\subsection{Resultados}

Fue medida la porosidad de las probetas. Los resultados se obtuvieron según:

$P_{1}=$ Peso de la probeta desecada a $105^{\circ} \mathrm{C}$ hasta pesada constante.

$P_{2}=$ Peso de la probeta saturada de agua.

$\mathrm{P}_{3}=$ Peso bajo agua, de la probeta saturada de agua $\left(P_{2}\right)$

\subsection{Resultats}

La porosité des éprouvettes a été mesurée; dont le résultat a été:

$P_{1}=$ Poids de l'éprouvette déssechées à $105^{\circ} \mathrm{C}$ - jusqu'à pesée constante.

$P_{2}=$ Poids de l'éprouvette saturée d'eau.

$P_{3}=$ Poids sous l'eau de l'éprouvette saturée d'eau. 
Llamamos porosidad al cociente entre el volumen de poros $\left(P_{2}-P_{1}\right)$ y el volumen aparente $P_{2}-P_{3}$.

$$
\text { Porosidad }=\frac{\left(P_{2}-P_{1}\right) 100}{P_{2}-P_{3}}
$$

Las intensidades relativas con anódo de cobre se midieron en
Nous appelons porosité le cotient entre le volume des pores ouverts, $\left(P_{2}-P_{1}\right)$ et le volume apparent, $P_{2}-P_{3}$ :

$$
\text { Porosite }=\frac{\left(P_{2}-P_{1}\right) 100}{P_{2}-P_{3}}
$$

Les intensivités relatives pour cuivre 1,54 $\AA$.

$$
\begin{aligned}
& \mathrm{CA} \text { en } 2 \theta=29,96^{\circ} \\
& \mathrm{C}_{3} \mathrm{AH}_{6} \text { en } 2 \theta=17,20^{\circ} \\
& \mathrm{AH}_{3} \text { en } 2 \theta=18,27^{\circ} \\
& \mathrm{CaCO}_{3} \text { en } 2 \theta=29,40^{\circ} \\
& \mathrm{CC}_{4} \mathrm{AH}_{12} \text { en } 2 \theta=11,64^{\circ} \\
& \mathrm{CAH}_{10} \text { en } 2 \theta=6,17^{\circ}
\end{aligned}
$$

Se midio el aumento de peso de las probetas que pesaban, de $595 \mathrm{~g}$ a $610 \mathrm{~g}$ y osciló de $0 \mathrm{a}$ 15 g como máximo.

Se analizó el contenido de $\mathrm{CO}_{2}$, la pérdida al fuego, el agua a $110^{\circ} \mathrm{C}$ y el agua combinada. Los datos obtenidos se pueden observar en la fig. 4.

En los tratamientos 9 (cúbico) y 10 (hexagonal), encontramos que en el 9 (cúbico), las resistencias a flexión y compresión y el contenido en $\mathrm{CA}$ disminuyen y aumentan los contenidos en $\mathrm{C}_{3} \mathrm{AH}_{6}$ y $\mathrm{AH}_{3}$. La disminución de las resistencias mecánicas de la conversión del hexagonal al cúbico puede compensarse por carbonatación como sucede en el caso 3.

En la fig. 4 se puede observar que el tratamiento 1 es insuficiente, por el bajo tiempo de carbonatación, ya que es grande su contenido de $\mathrm{C}_{3} \mathrm{AH}_{6}$ y bajo el de $\mathrm{CaCO}_{3}$. El tratamiento 2 no aumenta las resistencias mecanicas, ya que durante la segunda etapa del tratamiento la temperatura aumenta a $110^{\circ} \mathrm{C}$, la probeta al desecarse absorbe mal el $\mathrm{CO}_{2}$. Los compuestos producidos son semejantes a los del $1 .{ }^{\text {er }}$ caso.

El tratamiento 3 mejora las resistencias mecánicas, obteniéndose valores semejantes a los iniciales, del exagonal.

Con el mismo tiempo de carbonatación y una temperatura más baja $\left(20^{\circ}\right)$ en la segunda etapa. En el difractogramas disminuye el $\mathrm{C}_{3} \mathrm{AH}_{6}$ y aumenta el $\mathrm{CO}_{3}$ teniendo como resultado consiguiente un aumento del peso de la probeta.
L'augmentation du poids des éprouvettes fût mesuré; elles pesaient de l'ordre de 595 à 610 gr et oscillait de 0 à $15 \mathrm{gr}$ au maximum.

On été analysés: le contenu en $\mathrm{CO}_{2}$, la perte au feu, l'eau à $110^{\circ} \mathrm{C}$ et l'eau combinée, dont il n'y a pas de données, n'ayant pas trouve de corrélations. Les donnees obtenues sont représentées dans la fig. 4.

Lorsqu'on observe les résultats des traitements $9^{e}$ (cubique) et $10^{e}$ (Hexagonal), nous trouvons que dans le cas $d u$ n. ${ }^{\circ} 9$ cubique, les résistances à flexion et à compression et le contenu de CA diminuent et les teneurs en $\mathrm{C}_{3} \mathrm{AH}_{6}$ et en $\mathrm{AH}_{3}$ augmentent. La diminution des résistances mécaniques, de la conversion de l'exagonal au cubique, peut être compensées par carbonatation, come le traitement n. 3 indique.

Dans la fig. 4 on peut observer que le traitemen $n .^{\circ} 1$ est insuffisant en tant que temps de cargonatation, etant donné que la teneur en $\mathrm{C}_{3} \mathrm{AH}_{6}$ est grande et petite en $\mathrm{cC}$. Le traitement n. $2 n$ augmente pas les résistances mécaniques puisque, pendant la deuxième étape du traitement la température s'élève à $110^{\circ} \mathrm{C}$, l'éprouvette se dessèche et absorbe mal le $\mathrm{CO}_{2}$. Les característiques des composants produits sont similaires à celles du 1 er cas. Le traitement n. 3 améliore les résistances mécaniques atteignant des valeur pratiquement égales aux initiales, avec le même temps de carbonatation et une deuxième étape du traitement à une température plus basse $\left(20^{\circ}\right)$. Dans les difractogrammes on observe une descente du $\mathrm{C}_{3} \mathrm{AH}_{6}$, une élévation de la teneur en $\mathrm{CaCO}_{3}$, ayant comme resultat l'augmentation du poids de l'éprouvete. 
En el tratamiento 4, aumentó el tiempo de carbonatación ( 7 horas), disminuye sin embargo la resistencia a flexión; aumenta ligeramente la resistencia a compresión, y los contenidos de $\mathrm{CaCO}_{3}$ y $\mathrm{C}_{3} \mathrm{AH}_{6}$ bajan, lo cual indica que este tratamiento no es rentable comparado con el anterior. El tratamiento $5 \mathrm{da}$ la máxima cantidad de $\mathrm{CaCO}_{3}$, disminuye consecuentemente el $\mathrm{C}_{3} \mathrm{AH}_{6}$, que se descompone, aumenta la resistencia a la compresión y disminuye la resistencia a flexión. En este caso, el tiempo de carbonatación es menor que en el anterior. Las resistencias a flexión disminuyen y aumentan las resistencias y flexión disminuyen y aumentan las resistencias a compresión, cuando hay formación de carbonato cálcico.

Los tratamientos 6 y 7 , de larga duración (72 horas), llegan a mayores resistencias a compresión, pero con resistencias a flexión semejantes a las de la forma cúbica (tratamiento 9); de acuerdo con los difractogramas el $\mathrm{C}_{3} \mathrm{AH}_{6}$ desaparece cuando la temperatura de tratamiento con $\mathrm{CO}_{2}$ es de $85^{\circ} \mathrm{C}$, y queda un poco cuando el tratamiento con $\mathrm{CO}_{2}$ se realiza a $70^{\circ} \mathrm{C}$.

Finalmente, el tratamiento que da los mejores resultados de resistencia a compresión es el 8. En este caso la carbonatación dura 19 horas a $98^{\circ} \mathrm{C}$ con $\mathrm{CO}_{2}$ a $0,14 \mathrm{MPa}$ en medio húmedo, se sigue el tratamiento en medio húmedo $\sin \mathrm{CO}_{2}$, durante 43 horas a $110^{\circ} \mathrm{C}$. En este caso el aluminato monocálcico desaparece completamente y la formación de calcita es menor que en el caso anterior. La probeta tiene un aumento relativamente fuerte de peso (18 gramos) es decir un $30 \%$, hay formación de $\mathrm{C}_{3} \mathrm{AH}_{6}$ después de la carbonatación -con el CA que aún permanece del tratamiento con $\mathrm{CO}_{2}$, al calentar nuevamente a $110^{\circ} \mathrm{C}$, la probeta. La resistencia a compresión es notable, $90 \mathrm{MPa}$, aunque la resistencia a flexión desciende a $7,2 \mathrm{MPa}$, la porosidad del mortero es la más baja que se ha obtenido.

\subsection{Observaciones}

- Cuando las probetas se curan sumergidas en agua, desciende la resistencia a compresión y aumenta la resistencia a flexión. Esto que en la práctica no tiene utilización. Hay que tenerlo en cuenta para obtener resultados comparativos.

- El aumento de peso de las probetas es el método más simple y económico para comprobar el grado de carbonatación de las probetas; hay una relación con el
Avec le traitement n. 4 , le temps de carbonatation augment (7 heures), le résistance à flexión cependant diminue, la résistance à compression augmente légèrement, et la teneur en $\mathrm{CaCO}_{3}$ et $\mathrm{C}_{3} \mathrm{AH}_{6}$ s'abaisse, ce qui veut dire qu'il n'est pas rentable par rapport au précédent.

Le traitemen n. 5 donne le maximum de $\mathrm{CaCO}_{3}$, une diminution conséquence du $\mathrm{C}_{3} A \mathrm{H}_{6}$ qui se décompose, une augmentation de la résistance à compression et une diminution de la résistance à flexion. Dans ce cas, le temps de carbonatation est aussi inférieur au précédent. Les résistances à flexion diminuent et les résistances à compression augmentent lorsqu'il y a formation de carbonate calcique.

Les traitements n.os 6 et 7, très longs (72 heures), arivent à des résistances plus grandes à compresion mais avec des résistances à flexion semblables à celles de forme cubique qui, d'après les difractogrammes, disparaît lorsque la température du traitement avec $\mathrm{CO}_{2}$ est de $85^{\circ} \mathrm{C}$, et il en reste un peu lorsque le traitement avec $\mathrm{CO}_{2}$ est effectúe à $70^{\circ} \mathrm{C}$.

Finalement, le traitement qui a donné les meileurs resultats en tant que résistance à compression, c'esta le traitement n. ${ }^{\circ}$. Dans celui-là, la carbonatation s'effectue durant $19 \mathrm{~h}$ à $95^{\circ} \mathrm{C}$ avec $\mathrm{CO}_{2}$ à $0,14 \mathrm{MPa}$ en milieu humide, et le traitement se poursuit sans $\mathrm{CO}_{2}$ en milieu humide, pendant 43 heures, à $110^{\circ} \mathrm{C}$. Dans ce cas l'aluminate calcique disparaît complètement, et la formation de calcite est plus faible que dans le cas précédent; l'eprouvette à une augmentation de poids relatif très forte (18 grammes), c'est à dire $3 \%$ réel de l'augmentation du poids, il y a formation de quelque $\mathrm{C}_{3} \mathrm{AH}_{6}$ aprés la carbonatation - avec le CA qui restait avec le traitement avec $\mathrm{CO}_{2}$, en chauffant l'éprouvette à nouveau-. La résistance à compression est remarcable, 90 PMa, bien que la Flexion descende à 7,2 MPa; la porosité du mortier est la plus basse obtenue.

\subsection{Observations}

- Lorque les éprouvettes sont curées sous l'eau, la résistance à compression descend et la résistance à flexion augmente, ce qui, dans la pratique, n'est pas utilisable; mais il faut en tenir compte pour obtenir des résultats comparatifs.

- L'augmentation des poids dans les éprouvettes s'avère la méthode plus simple et plus économique à prouver le degré de carbonatation des éprouvettes, et il est en 


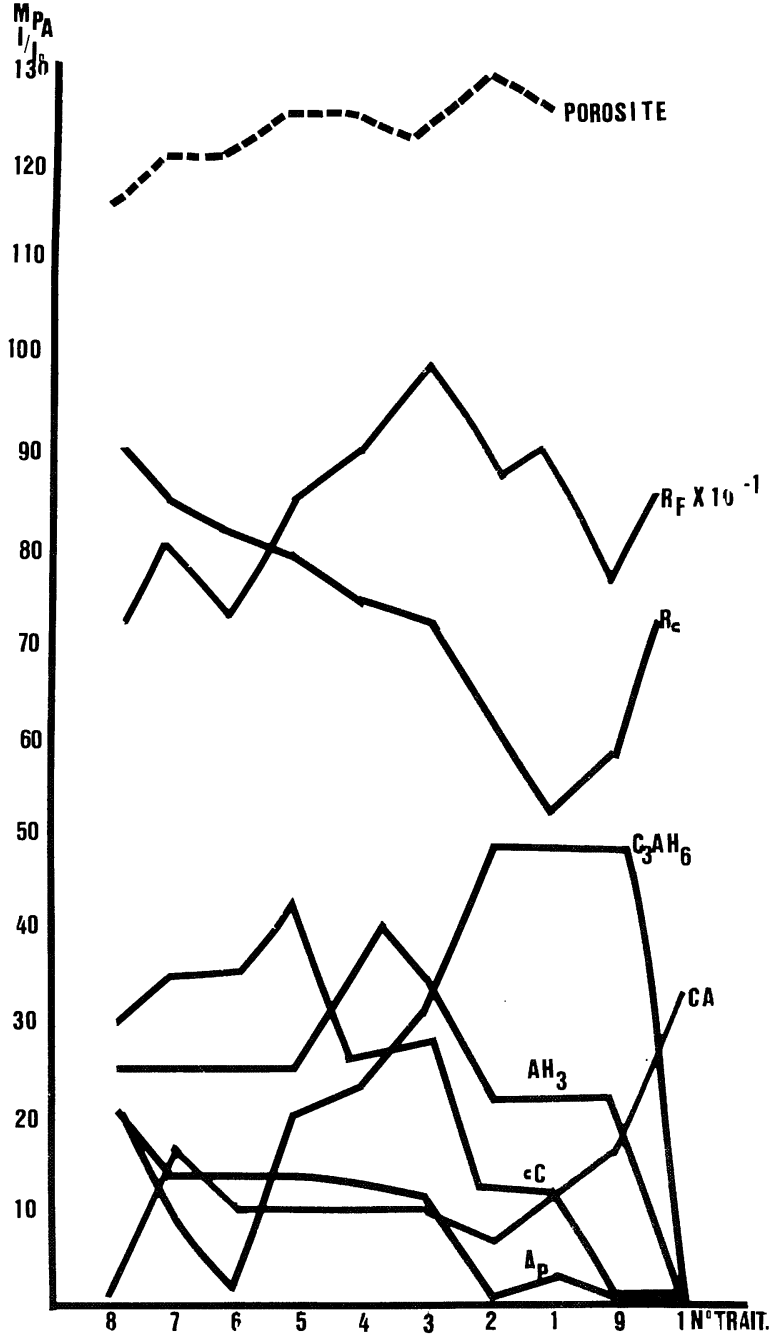

Fig. 4

aumento de las resistencias a compresión, ocasionada por la formación de calcita, aragonito $\mathrm{y} / \mathrm{o}$ vaterita.

- Durante la hidratación del cemento aluminoso a $20^{\circ} \mathrm{C}$, queda siempre una parte de aluminato monocálcico sin hidratar. En caliente a temperaturas elevadas $80^{\circ} \mathrm{C}-90^{\circ} \mathrm{C}$, con tiempo suficiente, se hidrata todo el aluminato monocálcico.

Si la carbonatación ha durado y sido suficiente, no debe de quedar aluminato tricálcico hexahidrato, ya que se debe de descomponer en aragonito, vaterita, calcita, alúmina $y / o$ hidróxidos de aluminio. Con el fin de encontrar las condiciones óptimas de tratamiento, en un tiempo razonable, se preparó una serie de probetas prismáticas de $4 \times 4 \times 16 \mathrm{~cm}$ con: $500 \mathrm{~g}$ de cemento aluminoso, $1.500 \mathrm{~g}$ de arena normalizada y

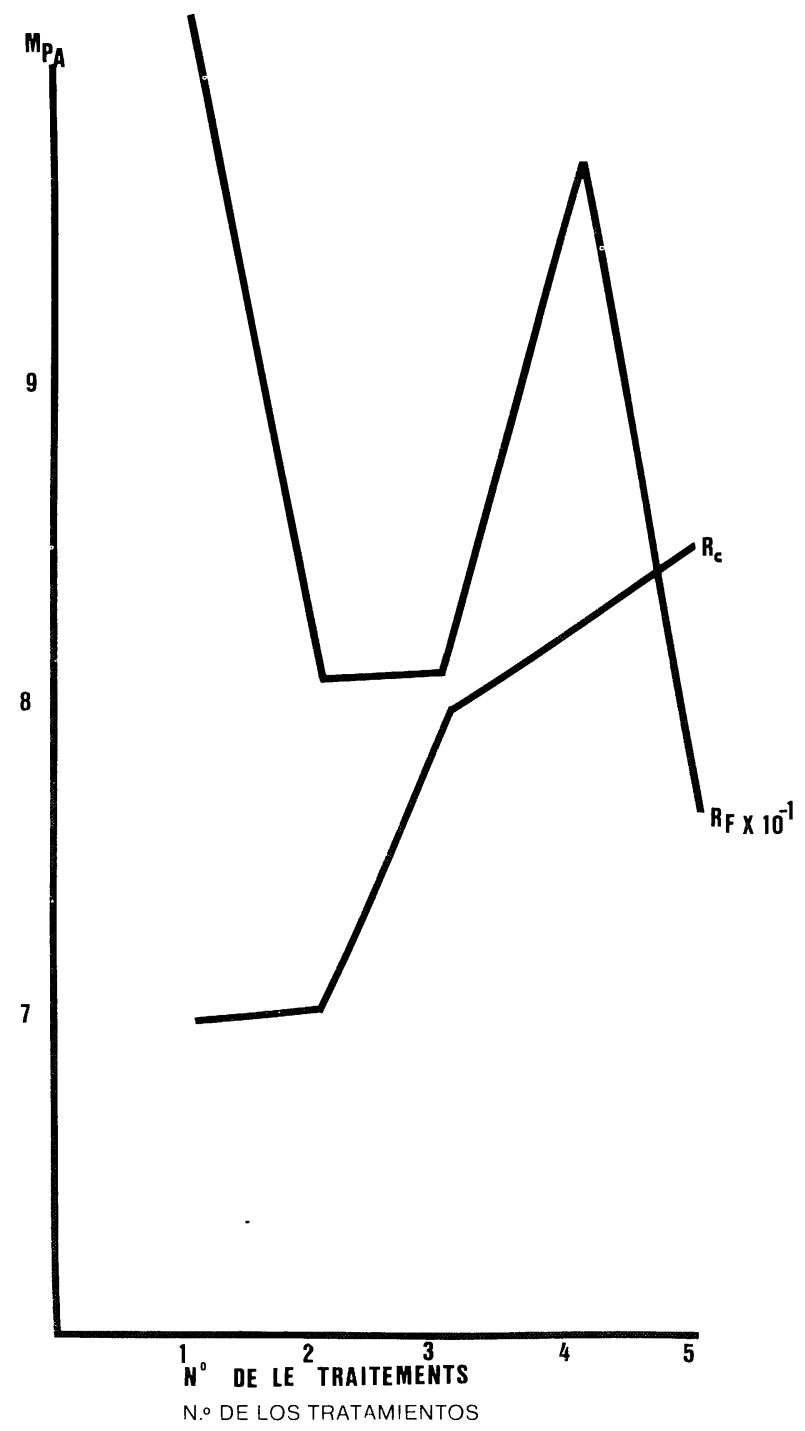

Fig. 5

relation avec l'augmentation des résistances à compression, étant donné que cette formation est due à la formation de calcite, aragonite et/ou vatérite.

- Pendant l'hydratation du ciment alumineux à $20^{\circ} \mathrm{C}$, une partie de l'aluminate calcique rest toujours sans s'hydrate. Si le temps d'hydratation au chaud a été suffisant, tout l'aluminate monocalcique est hydraté.

Si la carbonatation a duré et a été suffisante, il ne doit pas rester d'aluminate tricalcique hexahidraté car celui-là doit se décomposer, par carbonatation, en aragonite, vatérite, calcite, alumine et/ou hydroxides d'aluminium. A fin de trouver les conditions optimales du traitement, dans un temps raisonnable, une autre série d'éprouvettes prismatiques de $4 \times 4 \times 16 \mathrm{~cm}$ fût préparée avec: $500 \mathrm{gr}$ de ciment alumineux, $1.500 \mathrm{gr}$ de sable normalisé

MATERIALES DF COSTRUCCION. Vol. 37. n.²06. abril/mayo/junio 1987 
$220 \mathrm{ml}$ de agua desionizada. Los moldes de tres probetas, el cemento, arena y agua se calentaron a $100^{\circ} \mathrm{C}$. La temperatura de $100^{\circ} \mathrm{C}$ se mantuvo en el interior del recipiente a presión en donde estaba el molde, con las probetas durante 4 horas. Se desmoldearon las probetas que fueron sometidas a los tratamientos y curados siguientes.

1. 24 horas a $100^{\circ} \mathrm{C}$ con agua y $\mathrm{CO}_{2}$, curadas 24 horas bajo agua.

2. 28 horas a $20^{\circ} \mathrm{C}$ con agua y $\mathrm{CO}_{2}$, curadas 24 horas al aire.

3. 24 horas a $20^{\circ} \mathrm{C}$ con agua y $\mathrm{CO}_{2}$, curadas 24 horas al aire.

4. 24 horas a $100^{\circ} \mathrm{C}$ con agua y $\mathrm{CO}_{2}$, curadas 24 horas en cámara húmeda.

5. 24 horas a $100^{\circ} \mathrm{C}$ con agua y $\mathrm{CO}_{2}$, curadas 24 horas al aire.

Los resultados de las resistencias mecánicas a flexión y compresión se encuentran en la figura 5 .

Se observa el aumento de las resistencias a flexión cuando el curado se realiza bajo agua o con humedad a saturación.

En nuestra opinión el aumento de las resistencias mecánicas de $2 \mathrm{MPa}$, no se produce en la realidad, ya que las obras no se mantienen en condiciones de humedad a saturación o bajo agua (salvo canalizaciones, conducciones de agua y otras sumergidas en general), por lo cual estos resultados son ficticios.

La resistencia a compresión más elevada se da en los dos últimos tratamientos que implican un tiempo elevado de carbonatación de 24 horas.

La carbonatación puede ser efectuada industrialmente utilizando gases de combustibles que se pierden a menudo sin utilización ulterior en numerosas industrias; De la misma forma las temperaturas utilizadas pueden ser obtenidas con gases industriales entre $80^{\circ} \mathrm{C}$ y $100^{\circ} \mathrm{C}$ que se pierden en numerosos procesos, tales como fusión de hierro y otros metales, centrales térmicas, calefacción, fabricación de cementos, de hierro, de azucar, en general de todo proceso industrial que implique una combustión, sobre todo en los casos donde la recuperación de calor no se realiza con eficacia.

E! proceso en general implica una aceleración de los fenómenos que se dan de una forma natural en la hidratación del cemento aluminoso durante muchos años. Si se acelera et $220 \mathrm{ml}$ d'eau déionisée. Les moules des trois éprouvettes fût chauffé à $100^{\circ} \mathrm{C}$ ainsi que le ciment, l'eau et la sable. La température du moule des éprouvettes fût maintenue à l'intérieur du cuiseur pendant 4 heures. Elles furent ensuite démoulées et soumises aux traitements et aux cures suivants:

1. 24 heures à $100^{\circ} \mathrm{C}$ avec eau et $\mathrm{CO}_{2}$ curé 24 heures sous eau.

2. 28 heures à $20^{\circ} \mathrm{C}$ avec eau et $\mathrm{CO}_{2}$ curé 24 heures à l'air.

3. 24 heures à $20^{\circ} \mathrm{C}$ avec eau et $\mathrm{CO}_{2}$ curé 24 heures à l'air.

4. 24 heures $100^{\circ} \mathrm{C}$ avec eau et $\mathrm{CO}_{2}$ curé 24 heures chambre humide.

5. 24 heures $100^{\circ} \mathrm{C}$ avec eau et $\mathrm{CO}_{2}$ curé 24 heures à l'air.

Les résultats des résistances mécaniques à flexion et compression se trouvent dans la figure 5 .

On observe l'augmentation des résistances à fexion lorsque la curé est effectúe sous l'eau ou avec humidité à saturation. De toute façon notre opinion est que les augmentations de la résistance mécanique, de quelque $2 \mathrm{MPa}$ ne se produisent pasen réalité, car les ouvrages ne subissent pas ces conditions d'humidité, donc, les résultats sont fictifs. La résistance à compression la plus élevée se trouve dans les deux derniers traitements qui impliquent un temps élevé de 24 heures de carbonatation.

La carbonatation pourrait être effectuée industriellement en utilisant les gaz de combustion qui se perdent très souvent sans utilisation ultérieure dans de nombreuses industries; de même les températures employées peuvent être obtenues avec les gaz industriels entre $80^{\circ} \mathrm{C}$ et $100^{\circ} \mathrm{C}$ qui se perdent dans de nombreux procès, tes/s que fusions de fer et autres métaux, centrals thermiques, chauffages, fabrication de ciments, de fer, de sucre, en général de tout procès industriel qui comporte une combustion, surtout dans les cas òu la récupération de chaleur n'est pas effectuée avec efficacité.

Les procès, en géneral implique une accélération des phénomènes qui se sucèdent d'une façon naturalle dans l'hydratation di ciment aluminaux tout au long de plusieurs 
dicho proceso, se evitan las reacciones intermedias, con una economía del agua consumida en las reacciones, lo que beneficia a las resistencias mecánicas e impide su degradación. Hay una serie de reacciones, que aún siendo terminodinámicamente estables las fases formadas $\left(\mathrm{C}_{3} \mathrm{AH}_{6}\right)$, en contacto con el aire $\mathrm{y} / \mathrm{o}$ el $\mathrm{CO}_{2}$ dejan de serlo. Esto produce un aumento de las resistencias mecánicas y una disminución de las resistencias a flexión, pero siempre menores que las producidas por una conversión a través del aluminato cálcico decahidratado. années. Si on accélère cas procès, les réactions intermèdes sont évitées, avec une économie d'eau consumée, ce qui béneficie les résistances mécaniques et empêche leur dégradation. Il y a une série de réactions qui, même étant thermodinamiquement stables au contanct avec le gaz carbonique de l'air, cessent de l'être; ce qui produit une augmentation des résistance mécaniques et une diminution dans les résistances à flexion, mais toujours plus petites que celles produites par une conversion à travers l'aluminate calcique deca-hydraté.

\section{BIBLIOGRAPHIE}

1." On the carbonation mechanism of calcium aluminate hexahydrate in hydrated high alumina-cement. Supplementary paper Section III - III - 4 The VI International Congres on the chemistry of cement A. Ruiz de Gauna F. Triviño y T. Vázquez. Set. 1974. Moscow.

2. Estudio de las transformaciones del cemento aluminoso hidratado mediante las técnicas de difracción de Rayos $X$ Espectrometría Infrarroja y Análisis Térmico. Influencia del anhídrido carbónico, temperatura, humedad y adición de caliza en polvo. T. Vázquez, F. Triviño, A. Ruiz de Gauna. Materiales de Construcción n. 157 43-70. 1975 n. ${ }^{\circ} 158,1-48$. 1975. Monografía número 334. Julio 1976.

3. On the carbonation mechanism of calcium aluminato hexahydrate in hydrated hig alumina cement. Research working papers n. 31. F. Triviño, A. Ruiz de Gauna, T. Vázquez. Noviembre 1975. I.E.T c.c. (Madrid-33).

4. Nuevo procedimiento de fabricación de elementos prefabricados de hormigón de cemento aluminoso que no experimentan transformaciones perjudiciales. Patente n. 423.305 . C.S.I.C. España 1974.

5." Study of stabilized phases in high alumina cement mortars. M. Pérez, T. Vázquez, F. Triviño. Cement and Concrete Research. Vol. 13 759-770 1983. Vol. 14 1-10 - 1984.

6. Aspectos físico-químicos y estabilización de los aluminatos cálcicos resultantes de la hidratación del cemento aluminoso. M. Pérez Méndez, Fernando Triviño. Informes de la Construcción n. ${ }^{\circ} 332.1981$.

\section{publicación del i.e.t.c.c.}

\section{ACUEDUCTOS ROMANOS EN ESPAÑA \\ Carlos Fernández Casado \\ Prof. Dr. Ing. de Caminos, Canales y Puertos}

Esta publicación se compone de una serie de articulos, publicados en la Revista "Informes de la Construcción", en los cuales se hace un análisis de los acueductos romanos que existen en España y el balance de las condiciones de conservación en que se encuentra cada uno de ellos, incluyendo referencias históricas y literarias. Se ha ilustrado con la reproducción de la valiosa documentación gráfica que posee el prestigioso autor.

Un volumen encuadernado en couchè, a dos colores, de $21 \times 27$ centimetros, compuesto de 238 páginas, numerosos grabados, dibujos, fotos en blanco y negro y figuras de linea.

Precio: España, 900 ptas., 13 \$ USA.

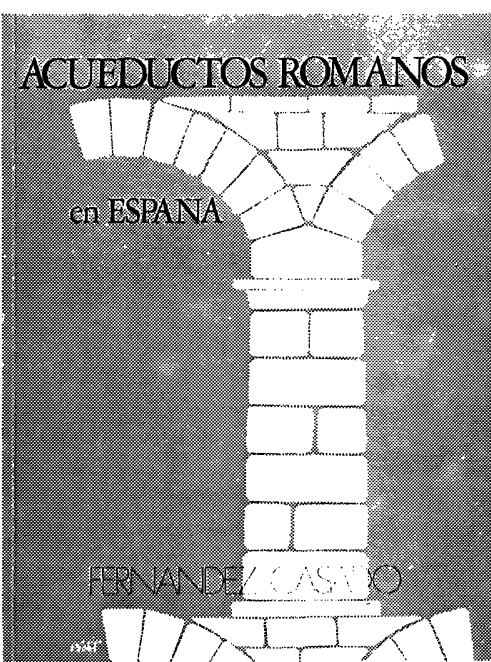

